Revue des patrimoines

33 | 2017

Patrimoine culturel immatériel et institutions patrimoniales

\title{
Des formes de continuité du patrimoine ethnologique : ethnologie des territoires, ethnologie du patrimoine, patrimoine culturel immatériel
}

About forms of continuity of the ethnological heritage: ethnology of territories, ethnology of heritage, intangible cultural heritage

\section{Christian Hottin}

\section{(2) OpenEdition}

\section{Journals}

Édition électronique

URL : http://journals.openedition.org/insitu/15469

DOI : 10.4000/insitu. 15469

ISSN : 1630-7305

Éditeur

Ministère de la culture

Référence électronique

Christian Hottin, « Des formes de continuité du patrimoine ethnologique : ethnologie des territoires, ethnologie du patrimoine, patrimoine culturel immatériel », In Situ [En ligne], 33 | 2017, mis en ligne le 02 novembre 2017, consulté le 02 mai 2019. URL : http://journals.openedition.org/insitu/15469 ; DOI : 10.4000/insitu. 15469

Ce document a été généré automatiquement le 2 mai 2019.

In Situ Revues des patrimoines est mis à disposition selon les termes de la licence Creative Commons Attribution - Pas d'Utilisation Commerciale - Pas de Modification 4.0 International. 


\section{Des formes de continuité du patrimoine ethnologique : ethnologie des territoires, ethnologie du patrimoine, patrimoine culturel immatériel}

About forms of continuity of the ethnological heritage: ethnology of territories, ethnology of heritage, intangible cultural heritage

\section{Christian Hottin}

\section{Qui porte le chapeau?}

1 «Au début des années 2000, alors que l'État prétendait vouloir se faire petit, déléguait ses compétences, transformait la déconcentration en décentralisation, la MPE [mission du Patrimoine ethnologique] cherchait un nouveau souffle et une nouvelle identité institutionnelle. À la faveur d'un toilettage de palais, elle reçut en 2003 une nouvelle dénomination: mission à l'Ethnologie. Normalisation disciplinaire? Trahison des espérances? Tactique de repositionnement dans la géographie du ministère? La question restait ouverte. Une chose était sûre: l'élimination de la référence au patrimoine ethnologique actait l'échec de la politisation de cet objet hybride. Restait pleine et entière la référence disciplinaire: l'ethnologie semblait s'imposer au patrimoine. Cependant, là où il y avait un dispositif bourdonnant d'activité, ne subsistait qu'un dispositif très diminué, perdu dans les longs couloirs feutrés des «Bons-Enfants». Que restait-il de la mission du Patrimoine ethnologique ? Une adresse Internet : http://www.culture.gouv.fr/mpe; «m-p-e», trois lettres qui permettaient d'ouvrir sur le site du ministère de la Culture et de la Communication la page de la mission à l'Ethnologie. 
Ce pastiche a été écrit en 2007, alors que nous lancions l'idée d'un regard rétrospectif sur une institution d'État, dont le changement de dénomination nous paraissait annoncer la disparition. Notre projet a pris un certain retard et a été dépassé par l'actualité administrative: au 31 décembre 2009, à la suite d'une réorganisation générale, la "mission Ethnologie » a disparu de l'organigramme du ministère de la Culture, même si, semble-t-il, il en subsiste les missions au sein $d u$ département du Pilotage de la recherche et de la Politique scientifique de la direction générale des Patrimoines. Au moins le lien est-il toujours actif... Quant aux satellites régionaux, leur nombre se réduit inexorablement sous l'effet d'une extinction naturelle du " corps ». " ${ }^{1}$

3 Rédigé entre 2007 (pour le premier paragraphe) et 2010 (pour le second), ce texte dont le titre général est La Disparition constitue la présentation d'un séminaire consacré à l'instauration du patrimoine ethnologique et organisé au sein du Laboratoire d'anthropologie et d'histoire de l'institution de la culture (LAHIC), avec le soutien de la mission Ethnologie, par Jean-Louis Tornatore et Noël Barbe, deux ethnologues fins connaisseurs de ce dispositif ${ }^{2}$, et qui en furent, ou en sont toujours, acteurs ${ }^{3}$. Le titre et la qualification de " pastiche » renvoient à une citation liminaire de Milan Kundera relatant la disparition, par l'action des services de propagande tchécoslovaques, de la figure de Vladimir Clementis sur une photographie où il se trouvait aux côtés de Klement Gottwald... Mais la toque qui coiffe Gottwald est celle de Clementis, prêtée par lui au premier : « De Clementis, il n'est resté que la toque de fourrure sur la tête de Gottwald. ${ }^{4}$ " De même, il ne serait resté du corps de la mission du Patrimoine ethnologique que l'adresse d'un site Internet, ultime remords, ou simple oubli, de l'administration'.

Dès lors, qui consulte aujourd'hui le site du ministère de la Culture et de la Communication pourra s'étonner d'y trouver une rubrique intitulée: "Patrimoine ethnologique ${ }^{6} »$, ainsi qu'une autre, qui répond quant à elle au titre de "Patrimoine culturel immatériel ${ }^{7} »$. Ce simple constat suggère deux séries de questions : d'une part, le patrimoine ethnologique ne serait donc pas mort, et quel sens peut prendre aujourd'hui cette expression? Et d'autre part, Le patrimoine culturel immatériel n'aurait pas, en définitive, remplacé le patrimoine ethnologique, contrairement à ce que laisse penser la suite du texte de présentation du séminaire ${ }^{8}$ ? Saisissant dans ce bref article la séquence 2006-2016, on se placera de part et d'autre de la révision générale des politiques publiques (RGPP), achevée en 2010, mais aussi entre l'approbation par la France de la convention pour la Sauvegarde du patrimoine culturel immatériel (loi 2006-791 du 5 juillet 2006) et l'inclusion de la définition du PCI dans le code du Patrimoine (loi 2016-925 du 7 juillet 2016). On tentera surtout, en manière de synthèse et de bilan, de comprendre comment, tout au long de ces dix ans, a été recherchée l'articulation entre une discipline (l'ethnologie) et une institution administrative (celle du Patrimoine).

\section{Principes généraux et organisation territoriale}

5 Le fil directeur du service en charge de l'ethnologie, au cours de ces dix années, a été de rechercher, d'abord au cas par cas, puis de plus en plus systématiquement, les axes de travail permettant d'ancrer l'ethnologie au sein de l'institution patrimoniale. À ce titre, l'ethnologie peut être insérée dans les politiques de l'institution de trois manières: soit en servant à qualifier et à étudier un patrimoine vu comme relevant de l'ethnologie (et c'est alors principalement le patrimoine culturel immatériel qui est concerné), soit en se saisissant de l'institution du patrimoine comme d'un objet d'étude (et l'on est alors dans le développement des recherches en ethnologie du patrimoine, telles que portées depuis 
2001 par le partenariat entre le ministère de la Culture, le CNRS et l'EHESS dans le cadre du LAHIC), soit en prenant la forme d'une ethnologie impliquée plus qu'appliquée, productrice d'effets sociaux et participant à la construction des territoires (et c'est alors le rôle des conseillers pour l'ethnologie au sein des directions régionales des Affaires culturelles qui est prédominant) 9 .

Ce principe directeur n'a pas été particulièrement affecté par les transformations liées à la RGPP et par ce qu'il faut bien appeler, s'agissant de l'ethnologie, la perte de nom. De ce point de vue, la perte du nom ne fut pas une perte du sens, ni un délitement des missions. Plus exactement, les effets négatifs de la perte du nom (absence de visibilité dans les organigrammes, déficit de lisibilité des missions par rapport à un nom de service affichant une mission généraliste transversale - département du Pilotage de la recherche et de la Politique scientifique), ont été en partie au moins compensés par des conséquences plus positives (réunion des publications de l'ethnologie et de la mission Ethnologie, qui étaient dissociées depuis 2005, réduction des circuits de décision par rapport à la hiérarchie administrative - chef du département et directeur général des Patrimoines). En revanche, l'absence de clarté quant aux lignes budgétaires affectées à l'ethnologie - vice de fonctionnement qui fut déjà cause de nombreux errements avant 2010 - perdure, et, de ce point de vue, l'émergence de la catégorie du "patrimoine culturel immatériel » n'a pas eu d'effet positif: le budget de l'ethnologie et du PCI demeure divisé entre trois programmes $(175,186$ et 224$)$ et peine à être distingué des lignes - beaucoup mieux dotées - allouées au patrimoine matériel.

Plus complexe s'est révélée, en revanche, l'organisation des réseaux de l'ethnologie sur le territoire national. Si l'on ne peut parler, s'agissant des conseillers pour l'ethnologie d'une "extinction naturelle du corps ${ }^{10}$ ", puisqu'un recrutement a été effectué en 2006 (Lorraine puis Rhône-Alpes), et un autre en 2014 (Guyane), ces renouvellements n'en restent pas moins très insuffisants pour combler les départs en retraite. Un des effets paradoxaux de la réforme des régions conduite pendant le quinquennat de François Hollande est que, en 2016, par le jeu des fusions, plus de territoires disposent d'un conseiller pour l'ethnologie que ce n'était le cas en 2010... (fig. 1) Mais se pose alors avec acuité la question de la nature du travail que peut conduire l'ethnologue : moins proche du terrain, tout en étant soumis, comme ses collègues des autres services, à des déplacements toujours plus fréquents. La mise en place dans certaines directions régionales de correspondants pour le patrimoine culturel immatériel, souvent très impliqués mais ne disposant pas de la même expertise, ne constitue pas, à l'évidence, une forme de continuité pour ce réseau. 


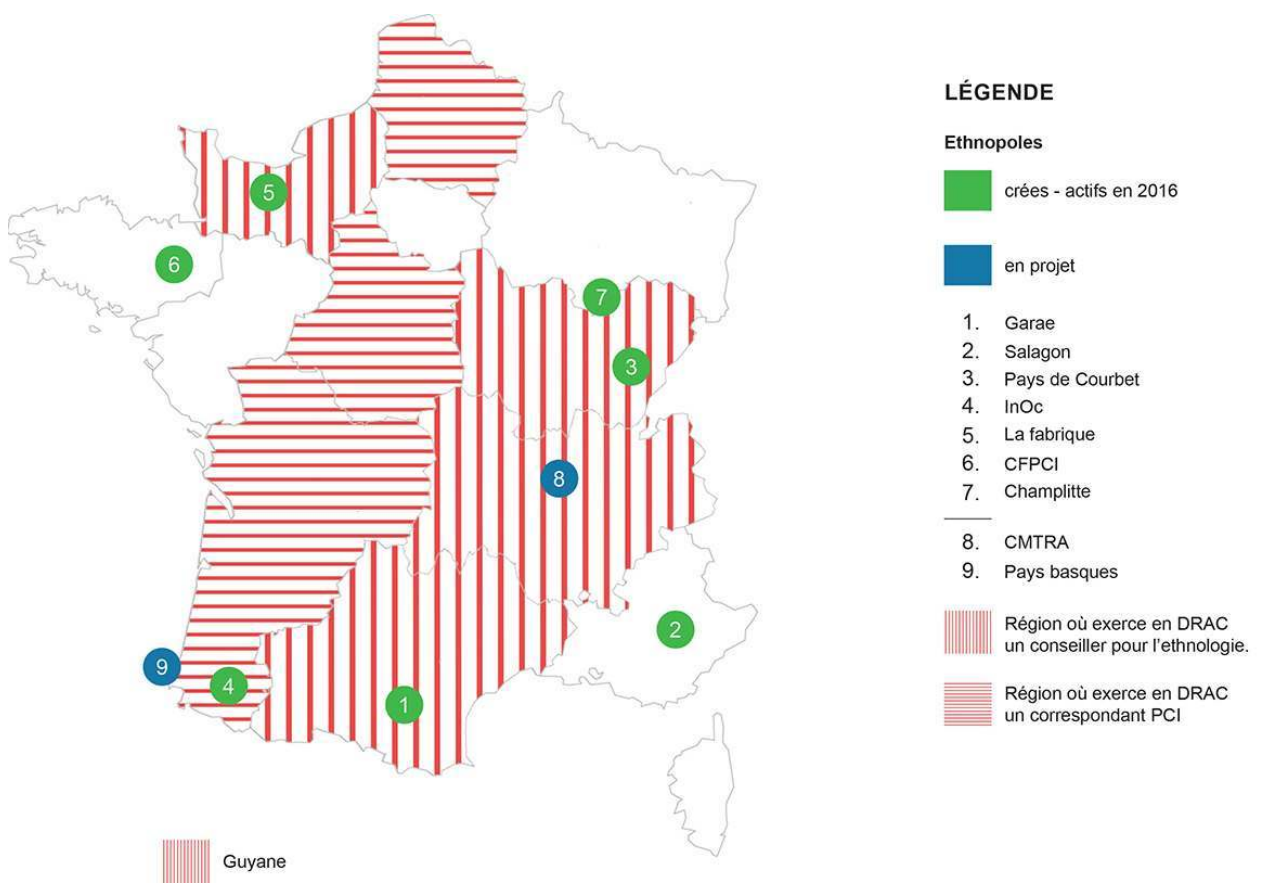

La répartition des conseillers pour l'ethnologie en DRAC et ethnopôles en 2016.

Conception et réalisation : Marianne Delaporte.

8 Le maillage territorial de l'ethnologie au ministère de la Culture a toujours été divers, en tout cas depuis la création en 1985 du Crécet (Centre régional de culture ethnologique et technique) à Caen, association portée par la région et par l'État et dont le directeur siégeait en tant qu'« ethnologue régional » aux réunions des chefs de service de la DRAC de Basse-Normandie. Ce maillage s'est enrichi à partir de 1996 d'un nouveau réseau, celui des ethnopôles ${ }^{11}$, Gérard Ermisse, chef de la mission du Patrimoine ethnologique étant le concepteur du dispositif et Christian Jacquelin, conseiller à la DRAC LanguedocRoussillon, l'inventeur heureusement inspiré du nom. Si le nombre de ces centres d'action culturelle et de recherches ethnologiques en région a longtemps stagné, se situant entre trois et quatre unités ${ }^{12}$, le réseau connait à partir de 2010 et surtout de 2014 une augmentation très sensible, pour atteindre le chiffre de sept membres en 2016, plus deux labellisations en projet. L'implication des conseillers pour l'ethnologie et des correspondants pour le PCI a été déterminante dans le processus de labellisation de plusieurs structures: Pays de Courbet, Institut occitan, musée des Arts et Traditions populaires de Champlitte, Institut culturel basque. La labellisation de la Fabrique des patrimoines en Normandie s'inscrit quant à elle dans la recomposition du paysage associatif patrimonial en Normandie, avec le regroupement autour du Crécet de différentes structures, tandis que celle du Centre français du PCI de Vitré s'inscrit dans le prolongement de la désignation par Frédéric Mitterrand du centre de documentation de la Maison des cultures du Monde à Vitré comme organisme de référence pour le PCI en France (disposition prévue à l'article 13 de la convention de l'Unesco du 17 octobre 2003). Ainsi les ethnopôles se sont-ils progressivement imposés comme structures jouant un rôle majeur dans le développement des réseaux de l'ethnologie en région, qu'ils soient ou non directement en relation avec la question du patrimoine culturel immatériel. 


\section{La recherche scientifique}

9

Le début des années 2000 marqua un tournant important de la recherche en ethnologie de la France au ministère de la Culture et de la Communication : la cessation d'activité du conseil du Patrimoine ethnologique et la création, d'abord en FRE (2000), puis sous forme d'UMR (2001), du LAHIC orientèrent de manière irréversible les thématiques de recherche portées par la Mission et les services qui lui succédèrent vers l'ethnologie du patrimoine. Encore que cette évolution s'avère moins linéaire qu'il n'y parait : entre 2000 et 2007, à l'instigation de Jean-Marie Jenn et de ses successeurs, se développa, sous la conduite de Bernard Haumont et Alain Morel, un important programme de recherche dédié aux formes de sociabilités dans l'habitat urbain, héritage, sans doute, d'une volonté de positionner l'ethnologie en situation stratégique d'expertise au sein d'une direction de l'Architecture et du Patrimoine ${ }^{13}$. De même, à partir de 2003, Odile Welfelé, chef de la mission à l'Ethnologie, lança un projet pluriannuel national de recensement des ressources ethnologiques, le PortEthno, qui, couplé à la mise en place du premier site Internet de la Mission, constitua une forme de développement enrichi du traditionnel Repethno, publié depuis les années 1980. Ces deux programmes se sont poursuivis jusque dans la deuxième moitié des années $2000^{14}$ et ont eu une incidence sur le travail de la mission Ethnologie au moment où elle commençait à orienter son action vers le patrimoine immatériel.

10 C'est toutefois bien l'ethnologie du patrimoine qui s'impose, à partir de 2006, comme le dénominateur commun de tous les programmes mis en place par le service de l'Ethnologie : «émotions patrimoniales » (2001-2009) ${ }^{15}$, «mémoires de l'immigration ", «vers un processus de patrimonialisation " $(2006-2011)^{16}$, « métiers du patrimoine " (2010-2016) ${ }^{17}$, «médiations du patrimoine» (depuis 2014) (fig. 2). Certains de ces programmes sont portés conjointement par le LAHIC et la mission Ethnologie ( $«$ les émotions patrimoniales », « les métiers du patrimoine »), d'autres le sont uniquement par le ministère, mais des chercheurs du laboratoire peuvent être conduits à y prendre part («mémoires de l'immigration»). Pourraient être ajoutés à cette liste différents séminaires, et notamment celui mis en place par Chiara Bortolotto et Sylvie Grenet sur le patrimoine culturel immatériel (2006-2009) ${ }^{18}$, qui témoignent de la coopération entre l'administration et les chercheurs sur ces questions et de la co-construction des problématiques de recherche. La collection des "Cahiers d'ethnologie de la France», série de la collection "Ethnologie de la France» dédiée aux ouvrages collectifs, a constitué le débouché naturel de ces travaux. Pour autant, comme on peut le constater, cette poursuite de l'activité dédiée aux programmes de recherche n'a pas entravé le lancement de l'Inventaire du patrimoine culturel immatériel, rendu obligatoire en vertu des articles 11 et 12 de la convention de l'Unesco : en fait, l'Inventaire du PCI en France, financé principalement par un appel à projets annuel, a progressivement pris la place occupée antérieurement par les enquêtes destinées à alimenter le PortEthno ${ }^{19}$. 


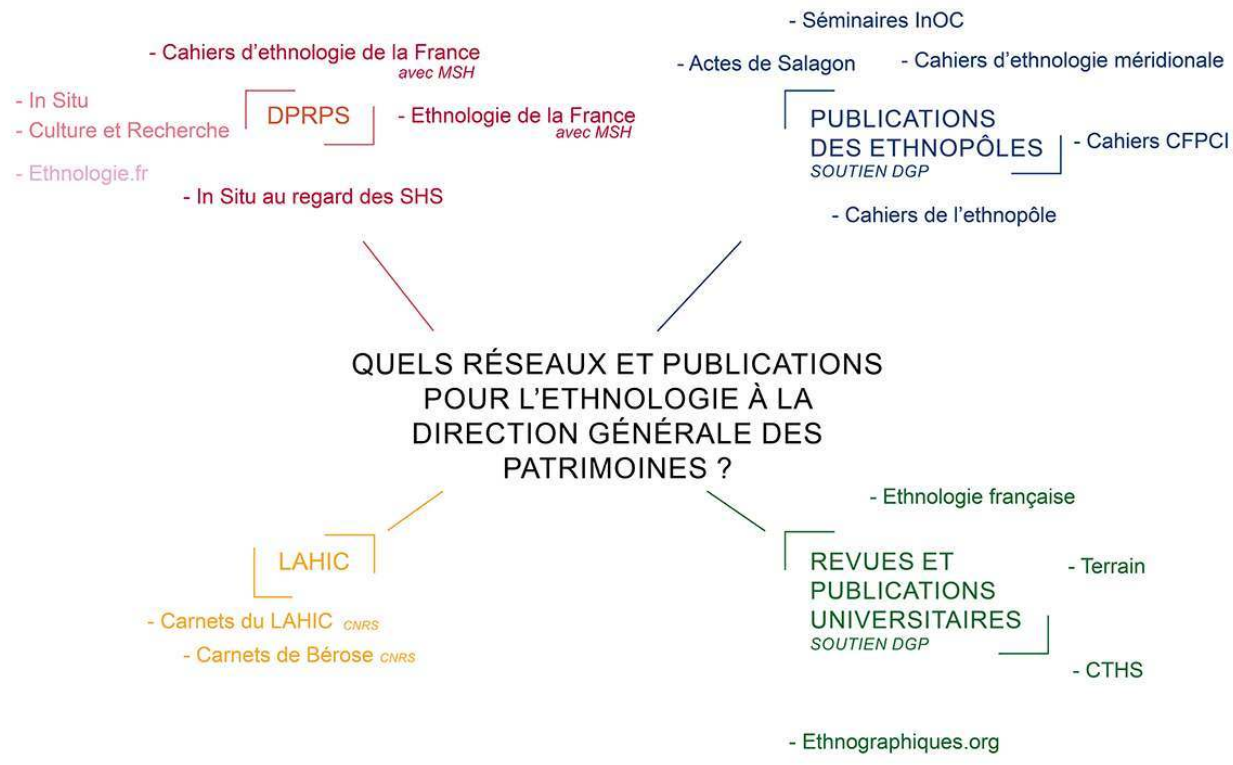

Les programmes de recherche en ethnologie de la France et patrimoine culturel immatériel pilotés par la mission du Patrimoine ethnologique et le DPRPS sur la période 2006-2016.

Conception et réalisation : Marianne Delaporte.

11 Domaine connexe de la recherche scientifique, la création audiovisuelle ${ }^{20}$ a fait l'objet d'un recentrage légèrement différent: soutenue par le biais d'un appel à projets annuel, la réalisation de documentaires en ethnologie de la France est restée jusqu'à ce jour ancrée dans une ligne beaucoup plus généraliste (aujourd'hui définie comme suit: « ethnologie de la France et patrimoine culturel immatériel »), quand bien même s'y retrouvent parfois des thématiques en rapport avec les politiques patrimoniales. En revanche, a été créée par Alain Morel, en partenariat avec la Huit production, une collection intitulée "L'ethnologie en héritage $~^{21}$ consacrée à des entretiens avec d'importantes figures de l'ethnologie française (et non nécessairement de la France). Riche de 18 titres, elle continue à se développer à raison d'un ou deux nouveaux portraits chaque année ${ }^{22}$. À l'évidence, cette collection se rattache plutôt à l'axe de travail "ethnologie du patrimoine", et même à sa composante "patrimoine et histoire de l'ethnologie » (principalement représentée par le programme Bérose au sein du LAHIC ${ }^{23}$.

\section{Vers une redéfinition de la stratégie des publications}

Les publications de l'ethnologie à la direction de l'Architecture et du Patrimoine étaient historiquement articulées sur trois modes de diffusion des résultats de la recherche : une collection de monographies (souvent issues de recherches individuelles financées par la Mission), une collection d'ouvrages collectifs (d'abord dédiée aux actes de colloques puis dévolue aux résultats des programmes de recherche) et enfin une revue, Terrain, d'abord centrée sur la restitution des programmes, puis progressivement toujours plus autonome jusqu'à avoir une ligne éditoriale totalement indépendante du reste de la politique en 
ethnologie de la France : ethnologie de l'Europe, voire internationale, et fort tropisme cognitiviste pour certains numéros. Pour autant, à partir des années 2000, grâce la mise en ligne de tous les numéros parus sur le portail revues.org, la revue est devenue la principale si ce n'est l'unique vitrine de l'action de l'ethnologie à la DAPA. Cette situation a perduré jusqu'à l'arrêt de la publication de la revue par le ministère, en 2015, et à la renaissance de celle-ci, au sein de la MAE de Nanterre, en 2016. Ces deux facteurs (grande visibilité de la revue et déconnexion de celle-ci par rapport à la politique de l'ethnologie) expliquent dans une certaine mesure la stratégie progressivement élaborée pour permettre au service de l'Ethnologie de donner une visibilité et une diffusion appropriée aux travaux élaborés en son sein ou avec son soutien, tout en rendant compte de la diversité des acteurs et des réseaux avec lesquels la Mission entretient des partenariats; l'objectif visé étant que les publications de l'Ethnologie, tout en étant aussi visibles que faire se peut, soient représentatives du travail effectué par le service au sein de l'administration du patrimoine (fig. 3).

Figure 3

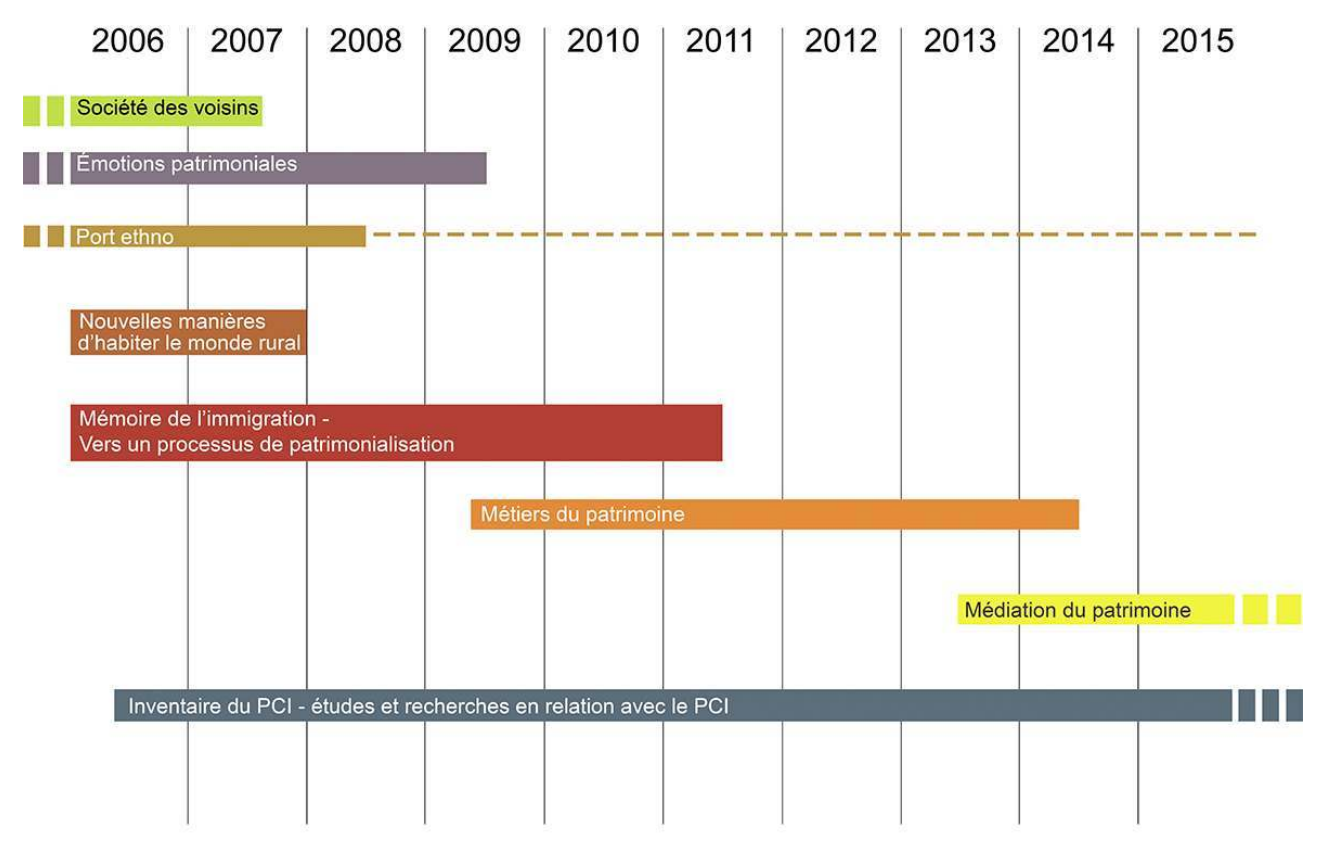

Les publications en ethnologie et patrimoine culturel immatériel et la direction générale des patrimoines en 2016.

Conception et réalisation : Marianne Delaporte.

13 À ce titre, un appui marqué a été donné aux publications des ethnopôles. En s'inspirant de la qualité et de la longévité des actes du séminaire d'ethnobotanique de Salagon ${ }^{24}$, issus des rencontres soutenues par la mission Ethnologie dans le cadre du partenariat avec l'ethnopôle, les établissements nouvellement labellisés ont été invités à faire figurer dans leur collaboration avec le ministère des collections de monographies ou d'actes de rencontres scientifiques : s'inscrivent dans cette perspective les "Cahiers de l'ethnopôle » (Pays de Courbet) ou les «Cahiers du CFPCI» (pour le Centre français du patrimoine culturel immatériel) ${ }^{25}$. Qu'il s'agisse des séminaires de Vitré (portant sur les politiques du $\mathrm{PCI}$ ), de ceux de Salagon (en ethnobotanique) ou des publications projetées par l'Institut 
occitan (en ethnomusicologie), la question du patrimoine culturel immatériel se trouve fréquemment présente dans ces publications.

Pour mettre en avant les publications réalisées dans le domaine de l'ethnologie du patrimoine, la mission Ethnologie a d'emblée soutenu et accompagné les projets portés par le LAHIC ( Carnets de Bérose ${ }^{26} »$, «Carnets du LAHIC $\left.{ }^{27} »\right)$, d'autant plus que ceux-ci permettaient une forme de complémentarité avec les collections historiques de la Mission. Il a ainsi été possible de faire paraitre sur un même sujet (les émotions patrimoniales) un ouvrage collectif dans la collection des «Cahiers d'ethnologie de la France» et trois monographies de terrain publiées in-extenso dans la collection des " carnets ${ }^{28}$, tandis qu'une seule et même recherche - l'enquête de Nathalie Heinich sur l'Inventaire général du patrimoine - se déploie en une monographie et un recueil d'articles, la première dans la collection «Ethnologie de la France ${ }^{29}$ ", le second dans les "Carnets du LAHIC ${ }^{30}$ ».

15 Avec l'arrêt de la publication de Terrain par le ministère et sa "renaissance ${ }^{31}$ » à la MAE de Nanterre, les deux collections publiées en partenariat avec la Maison des sciences de l'Homme sont apparues plus que jamais au cœur de la ligne scientifique et éditoriale de l'ethnologie au sein de la direction des Patrimoines. Néanmoins, cette affirmation de la place de l'ethnologie au sein de l'administration passe aussi par sa présence dans d'autres publications : la revue Culture et recherche, éditée au sein du Secrétariat général et très largement diffusée ${ }^{32}$, ou bien entendu, la revue In Situ, qui fut à l'origine la revue en ligne de l'Inventaire général mais s'est progressivement ouverte à l'ensemble des domaines et disciplines patrimoniaux. Cette présence de l'ethnologie devrait être plus importante encore grâce à la mise en place prochaine d'une série d'In Situ dédiée aux sciences sociales.

Enfin, à partir d'un cœur de ligne éditoriale solidement ancré sur le patrimoine, il est possible de nouer des partenariats avec des publications travaillant à la diffusion des résultats de la recherche généraliste en ethnologie : celles-ci, liées au monde universitaire et n'ayant pas forcément vocation à faire du patrimoine leur objet propre, peuvent construire néanmoins avec le service de l'Ethnologie au ministère des partenariats durables, qui visent à l'animation de la recherche scientifique. C'est dans cet esprit qu'ont été progressivement nouées des relations avec ethnographiques.org, Ethnologie française, le Comité des travaux historiques et scientifiques (CTHS), ou encore Terrain (depuis sa refondation à Nanterre).

17 Là encore, comme on le voit, la politique de mise en œuvre de la convention sur le patrimoine culturel immatériel ne s'est pas substituée à la politique du patrimoine ethnologique: elle s'inscrit, à travers un soutien marqué à quelques titres, dans une action plus générale en faveur des publications, qui fait la part belle aux travaux en ethnologie du patrimoine ${ }^{33}$ sans pour autant se détourner d'un accompagnement des publication généralistes qui font référence en matière de recherche ethnologique. Si la politique en faveur du PCI ne se confond ni avec les réseaux territoriaux de l'ethnologie, ni avec ses thématiques de recherche, ni avec sa politique de publication, il importe désormais de voir comment elle se manifeste, et comment elle travaille avec l'ethnologie. Comme on le verra, ce travail est possible, encore qu'il n'aille pas sans ajustements critiques. 


\section{Le PCl sous le signe de l'ethnologie : inventaires, inscriptions, réseaux ${ }^{34}$}

Confiée dès 2006 à la mission Ethnologie, la mise en œuvre de la convention pour la Sauvegarde du PCI a d'emblée été placée sous le signe de l'ethnologie. Cette alliance entre une discipline scientifique et une catégorie patrimoniale a constitué la clef de voûte de cette politique. Bien des raisons plaident en faveur d'un tel parti : outre que la plupart des politiques patrimoniales reposent sur de pareilles unions, la définition du patrimoine ethnologique, posée en 1980 dans le rapport Benzaïd ${ }^{35}$, annonçait largement celle du PCI selon l'Unesco, et ce rôle pionnier de la France en matière d'ethnologie avait été rappelé par d'éminents conseillers d'État en 2005 lors des discussions préparatoires à la ratification. L'implication de la communauté des ethnologues dans la sauvegarde du PCI ne va toutefois pas de soi, et, si nombreux sont ceux qui s'y sont attachés dès le commencement, le PCI continue de susciter des réactions de rejet chez des chercheurs de premier plan $^{36}$.

19 Ce rapprochement a eu comme première conséquence de détourner la Mission, alors accoutumée depuis près de dix ans, comme on l'a vu, aux problématiques de l'ethnologie du patrimoine, d'une mise en œuvre immédiate de la convention. De fait, les premières actions conduites, les premiers financements octroyés le furent en vue d'étudier le PCI comme politique, et non dans le but de mener des actions découlant directement de la mise en œuvre de la convention. Ce furent les projets de recherche de Gaetano Ciarcia ${ }^{37}$, les séminaires de Chiara Bortolotto et Sylvie Grenet ${ }^{38}$, et différents colloques, jusqu'à celui tenu en 2012 à Cerisy-la-Salle. Dès l'origine, la mission Ethnologie a souhaité placer son action dans le domaine du PCI sous le signe du partage de l'expérience avec les chercheurs et a accepté que son action devienne elle-même objet d'étude, voire de critique, pour les anthropologues. Inauguré sous le regard des ethnologues, ce partenariat se poursuit avec des chercheurs de différentes disciplines, notamment des juristes ${ }^{39}$ ou des économistes ${ }^{40}$ et est toujours extrêmement fécond.

Les critiques, justement, n'ont pas été rares lorsque s'est engagé, en 2007, l'Inventaire du $\mathrm{PCI}$ en France. L'idée même d'un tel inventaire fut alors critiquée par plusieurs chercheurs ${ }^{41}$. De fait, il est sans doute l'aspect de la mise en œuvre de la convention qui a le plus évolué en quelques années. Conçu au départ selon une double perspective (répertorier les instruments de recherche existants et mettre en place un inventaire des pratiques vivantes), il a conservé jusqu'à aujourd'hui ce caractère mixte, mais son mode de constitution n'est plus, comme en 2007, centré sur la réalisation d'enquêtes d'inspiration ethnographique. En vue d'une prise en compte toujours plus large de la participation des praticiens, il est nourri par un appel à projets annuel et par des candidatures spontanées, qui servent souvent de tremplin à des dossiers pour l'Unesco, tout en constituant, de plus en plus souvent, une forme de reconnaissance en soi. L'Inventaire du PCI compte aujourd'hui plus de 350 entrées, et les appels à projets annuels ont permis la conduite de 33 opérations d'inventaire.

21 Encore que cela puisse sembler moins évident, cette relation privilégiée à l'ethnologie a été essentielle dans les candidatures pour l'Unesco. L'absence de visibilité initiale du PCI a eu pour conséquence que les candidatures sont rapidement apparues comme un moyen de développer l'intérêt pour cette politique. Non sans succès. Avec l'appui de la direction générale des Patrimoines, des ethnologues des DRAC et de plusieurs chercheurs 
impliqués, en l'espace de huit ans, quinze éléments du PCI français ont été inscrits, principalement sur la liste représentative. Deux autres dossiers sont déposés et le service en charge de l'Ethnologie a eu à connaître plus de cent dix projets de candidatures, dont un nombre important est toujours en cours. Seuls trois dossiers ont échoué devant le comité intergouvernemental. Si l'on excepte le cas atypique du repas gastronomique des Français, le tableau d'ensemble des éléments inscrits fait ressortir le refus de l'exceptionnel, l'importance des pratiques modestes, parfois peu spectaculaires (savoirfaire de la dentelle d'Alençon), fortement portées par des communautés groupées en association (fest-noz, Gwoka), ayant souvent fait l'objet antérieurement de recherches ethnologiques (trait de charpente, compagnonnage). Le portage des dossiers par les détenteurs de $\mathrm{PCI}$ est la règle, la qualité du plan de sauvegarde et la participation des communautés les principaux critères d'évaluation. Il en va de même pour différents projets en cours (fondation de l'œeuvre Notre-Dame à Strasbourg, fêtes de l'Ours, Pourimshpiel). Les dossiers français sont régulièrement salués par le comité intergouvernemental pour leur grande qualité. Très loin d'une politique de labellisation à courte vue, qui n'aboutirait qu'à faire de ces listes des "villages Potemkine ", plusieurs porteurs de projet ont tenu à associer ces candidatures à une prise en compte du PCI au sein des politiques culturelles ou de développement local (fest-noz, tapisserie d'Aubusson) ${ }^{42}$.

Opérations d'inventaire et candidatures sont indissociables d'un très important travail de construction et de dynamisation des réseaux liés au PCI, qui sont aussi souvent ceux de l'ethnologie associative ou des territoires. Ce travail s'est appuyé sur des réseaux fédératifs (Fédération des associations de musiques et danses traditionnelles, FAMDT, Fédération des écomusées et des musées de société, FEMS), et sur des maillages territoriaux (tout particulièrement en Bretagne). Dans un contexte politique où la notion de communauté n'est pas clairement acceptée, les réseaux associatifs des porteurs de traditions sont des relais puissants pour mener des opérations d'inventaire, porter des candidatures, sensibiliser les élus et organiser des événements culturels et scientifiques (plus de 90 en dix ans). Depuis 2011, la création sous l'égide de la Maison des cultures du monde du Centre français du PCI à Vitré, labellisé ethnopôle en 2015, a permis un réel essor de ces réseaux et une meilleure coordination: réseau des vingt ONG françaises accréditées auprès de l'Unesco, des porteurs d'éléments inscrits sur les listes (association France-PCI) et réseaux de chercheurs et professionnels européens qui prennent part au séminaire annuel soutenu par la direction des Patrimoines. Enfin, opérations d'inventaire, candidatures, projets de recherche-action aboutissent à plusieurs mises en valeur de l'attachement au PCI. Différentes expositions récentes témoignent de l'important potentiel qu'offre le PCI en matière de médiation: "Sortez des clichés!", réalisée par la FEMS ${ }^{43}$, « (Ex)traordinaire quotidien », conçue par l'université de Pau avec l'ethnopôle InOc et présentée au musée d'Ethnographie de l'Université de Bordeaux ${ }^{44}$, et enfin « Exposez votre PCI en Alsace Bossue »"

Au-delà des candidatures, de l'Inventaire et des opérations de recherche, l'implication du service en charge de l'Ethnologie (mission ou département) se déploie sur l'ensemble du domaine des politiques du PCI, depuis les enjeux législatifs les plus forts (inclusion du PCI dans la loi LCAP, par exemple), jusqu'aux actions en faveur de la valorisation de ce même patrimoine (dotation de prix dans des festivals) (fig. 4). 


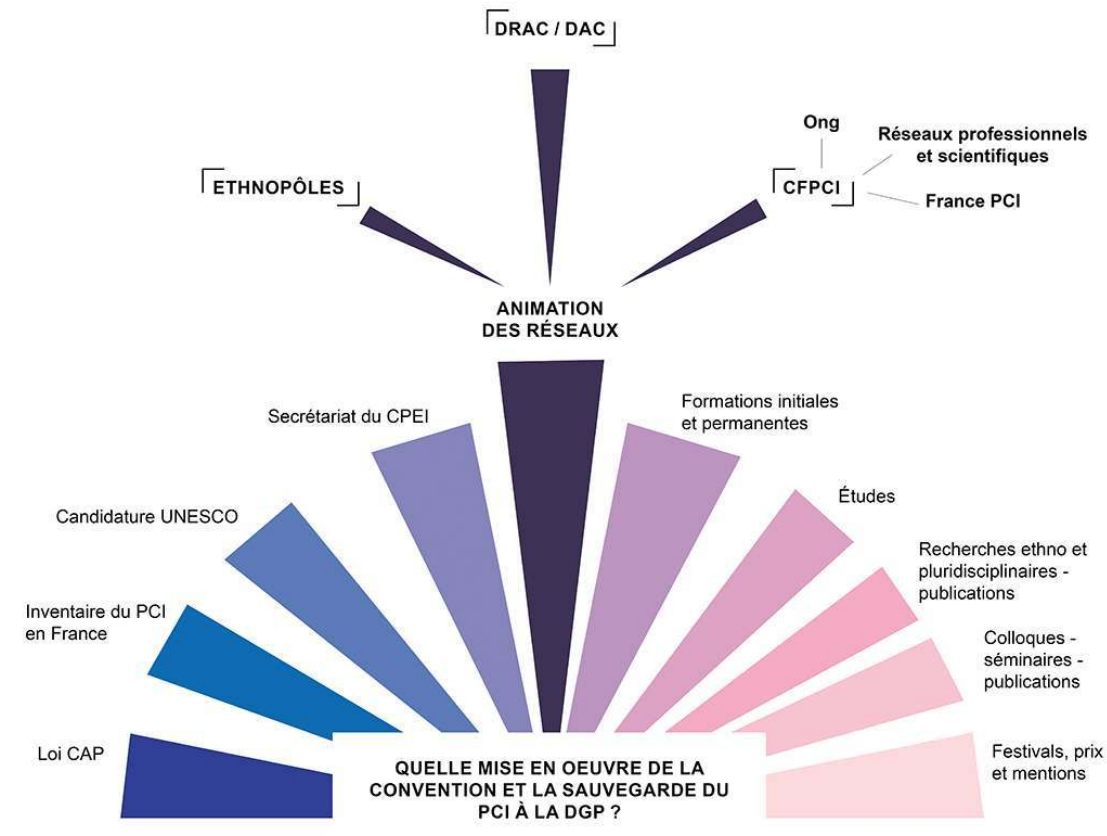

Les missions et actions du DPRPS dans le domaine du patrimoine culturel immatériel en 2016.

Conception et réalisation : Marianne Delaporte.

En définitive, le patrimoine culturel immatériel ne s'est pas substitué au patrimoine ethnologique : la politique menée en application de la convention de l'Unesco pour la Sauvegarde du PCI - ou ce que l'on a appelé « la prise française du patrimoine culturel immatériel » - ne constitue que l'une des modalités de mise en œuvre de la politique en ethnologie de la France, selon une articulation assez complexe: la question du PCI innerve les autres domaines d'action du ministère sans les recouvrir totalement (ethnologie des territoires, ethnologie du patrimoine, recherches, publications), et, en même temps, à travers ces outils spécifiques que sont les candidatures et l'Inventaire du PCI en France, le PCI est un domaine en propre de l'action ministérielle. Ainsi, il constitue une modalité plus pratique et plus opérationnelle de la politique en ethnologie de la France : fortement ancrée dans le droit (elle peut se prévaloir de la mise en œuvre d'un traité, catégorie d'outils juridiques située au plus haut de la hiérarchie des normes) et axée sur la recherche de la sauvegarde ${ }^{46}$ (et donc, en cela, compatible avec les objectifs généraux de l'institution patrimoniale, tels que les textes les définissent) : la direction générale des Patrimoines « est chargée de l'étude, de la protection, de la conservation, de la restauration, de la valorisation et de la transmission aux générations futures du patrimoine et des collections des musées, du patrimoine archéologique, des monuments et des espaces protégés, ainsi que des autres biens culturels, protégés au titre du code du Patrimoine et du code de l'Urbanisme pour leur intérêt historique, esthétique et culturel. Elle exerce ces mêmes compétences au titre des œuvres photographiques et du patrimoine ethnologique et immatériel ${ }^{47}$.» 


\section{NOTES}

1. - TORNATORE, Jean-Louis et BARBE, Noël. "Séminaire. L'instauration du patrimoine ethnologique. Texte de présentation » [publié le 22 novembre 2010]. Disponible en ligne sur le site du IIAC (LAHIC) : http://www.iiac.cnrs.fr/article887.html [consulté le 24/10/2017].

2. - Voir : BARBE, Noël. «Isac Chiva, ethnologie et politique patrimoniale ». Terrain [En ligne], 60| mars 2013, mis en ligne le 19 août 2014, consulté le 22 décembre 2016. URL: http:// terrain.revues.org/15127 ; et TORNATORE, Jean-Louis. «La difficile politisation du patrimoine ethnologique ». Terrain [En ligne], 42 | mars 2004, mis en ligne le 9 septembre 2008, consulté le 22 décembre 2016. URL : http://terrain.revues.org/1791.

3. - Ce séminaire a trouvé un prolongement avec la tenue, en décembre 2016, d'un colloque intitulé «Du moment du patrimoine ethnologique», organisé en partenariat avec l'Institut interdisciplinaire d'anthropologie du contemporain (IIAC), avec le soutien de la direction régionale des Affaires culturelles de Bourgogne-Franche-Comté. Voir le site: http:// www.iiac.cnrs.fr/article2900.html [consulté le 24/10/2017].

4. - KUNDERA, Milan. Le Livre du rire et de l'oubli [1978]. Paris : Gallimard, 1985, p. 14.

5. - Internet étant un grand cimetière d'astres morts mais toujours visibles, le site est toujours accessible, près de sept ans après la réorganisation de l'administration centrale: http:// www.culture.gouv.fr/mpe/.

6. - Elle figure dans l'onglet $d u$ site dédié aux politiques ministérielles: http:// www.culturecommunication.gouv.fr/Politiques-ministerielles/Patrimoine-ethnologique.

7. - Elle figure dans l'onglet $d u$ site dédié aux politiques ministérielles: http:// www.culturecommunication.gouv.fr/Politiques-ministerielles/Patrimoine-culturel-immateriel.

8. - «En aval, ce paradoxe est pour ainsi dire digéré: le patrimoine ethnologique trouve aujourd'hui une résolution politiquement consensuelle et un prolongement "naturel", quoique problématique, dans la catégorie, internationalement reconnue, de patrimoine culturel immatériel ». TORNATORE, Jean-Louis et BARBE, Noël. « Séminaire. L'instauration du patrimoine ethnologique. Texte de présentation » [publié le 22 novembre 2010]. Disponible en ligne sur le site du IIAC (LAHIC) : http://www.iiac.cnrs.fr/article887.html [consulté le 24/10/2017].

9. - Ces trois axes ont été largement traités sous la forme d'études de cas particulières et d'analyses plus générales dans le cadre d'un dossier de la revue Culture et recherche coordonné par Christian Hottin, Marina Chauliac et Noël Barbe, «Les nouveaux terrains de l'ethnologie », $\mathrm{n}^{\circ}$ 127, automne 2012. Disponible en ligne sur le site du ministère de la Culture: http:// www.culturecommunication.gouv.fr/Politiques-ministerielles/Recherche-Enseignementsuperieur-Technologie/La-revue-Culture-et-Recherche/Les-nouveaux-terrains-de-l-ethnologie. 10. - TORNATORE, Jean-Louis et BARBE, Noël. «Séminaire. L'instauration du patrimoine ethnologique. Texte de présentation" [publié le 22 novembre 2010]. Disponible en ligne sur le site du IIAC (LAHIC): http://www.iiac.cnrs.fr/article887.html [consulté le 24/10/2017]. L'extinction du corps est d'autant moins «naturelle » qu'elle apparaît causée avant tout par le départ, au fil des ans, de nombreux conseillers vers d'autres corps de fonctionnaires (maîtres de conférences, chargés de recherche, conservateurs du patrimoine) ou vers des positions les mettant en retrait de l'action administrative (mise à disposition au CNRS). Une telle volatilité probablement sans équivalent dans les corps scientifiques ou de recherche du ministère de la Culture - est tout sauf naturelle et pose question.

11. - «L'appellation ethnopôle s'attache à une institution qui, en matière de recherche, d'information et d'action culturelle, œuvre à la fois au plan local et au niveau national. À travers 
cette appellation, la mission du Patrimoine ethnologique entend, dans le cadre propre à chaque structure, promouvoir une réflexion de haut niveau s'inscrivant tout à la fois dans les grands axes de développement de la discipline ethnologique et dans une politique de constitution des bases d'une action culturelle concertée " (rapport Mission ethnopôles, 1999). Voir le rapport rédigé en 1999 sur le fonctionnement de ce réseau: DUBOST, François, LIZET, Bernadette et ZONABEND, Françoise. Mission ethnopôles, rapport au conseil du patrimoine ethnologique. Paris: mission du Patrimoine ethnologique, 1999, disponible en ligne: http:// www.culturecommunication.gouv.fr/Politiques-ministerielles/Patrimoine-ethnologique/ Patrimoine-ethnologique2/Ethnologues-en-region/Ethnopoles.

12. - De quatre à l'origine (GARAE, musée-conservatoire de Salagon, Maison du Fleuve Rhône et Estuarium), le nombre d'ethnopôles chute durablement à trois après la disparition de l'association Estuarium en 2006.

13. - HAUMONT, Bernard et MOREL, Alain (dir.). La Société des voisins. Partager un habitat collectif. Paris : Éd. de la MSH, coll. «Cahiers d'ethnologie de la France », 2005. Disponible en ligne : http:// books.openedition.org/editionsmsh/3347 [consulté le 24/10/2017].

14. - L'ouvrage conclusif du programme de recherche fut publié en 2005, mais un colloque prenant en compte les recherches menées lors d'une seconde vague de recherches se tint en décembre 2007.

15. - FABRE, Daniel (dir.). Émotions patrimoniales. Paris : Éd. de la MSH, coll. « Cahiers d'ethnologie de la France ", 2013. Disponible en ligne: http://books.openedition.org/editionsmsh/3580 [consulté le 24/10/2017].

16. - BARBE, Noël, CHAULIAC, Marina (dir.). L'Immigration aux frontières du patrimoine. Paris: Éd. de la MSH, coll. « Cahiers d'ethnologie de la France », 2014.

17. - HOTTIN, Christian, VOISENAT, Claudie (dir.). Le Tournant patrimonial. Mutations contemporaines des métiers du patrimoine. Paris : Éd. de la FMSH, coll. «Cahiers d'ethnologie de la France ", 2016.

18. - BORTOLOTTO, Chiara (dir.). Le Patrimoine culturel immatériel. Enjeux d'une nouvelle catégorie. Paris : Éd. de la MSH, coll. « Cahiers d'ethnologie de la France », 2011. Disponible en ligne : http:// books.openedition.org/editionsmsh/3545 [consulté le 24/10/2017].

19. - PortEthno fait toutefois l'objet de mises à jour régulières $(2012,2016)$ et une nouvelle rubrique y a été créée en 2015, dédiée aux sources d'archives de l'ethnologie de la France.

20. - Il est à noter que cette question du soutien à la création audiovisuelle, pourtant constitutive de la politique du patrimoine ethnologique est restée totalement «hors-champ» dans le séminaire consacré à la MPE.

21. - Voir le site: http://www.lahuit.com/fr/la-boutique/grands-entretiens [consulté le 24/10/2017].

22. - Le dernier en date, consacré à Daniel Fabre, a été réalisé peu de temps avant la disparition de l'anthropologue: http://www.lahuit.com/fr/content/daniel-fabre [consulté le 24/10/2017]. Les entretiens à venir seront consacrés à Martine Segalen et Françoise Zonabend.

23. - L'encyclopédie en ligne sur l'histoire des savoirs ethnographiques est disponible à l'adresse suivante : www.berose.fr [consulté le 24/10/2017].

24. - La liste complète des actes du séminaire est disponible en ligne : http://www.musee-desalagon.com/la-recherche/les-publications.html [consulté le 24/10/2017].

25. - Les ouvrages de cette collection sont disponibles en ligne sur le site du CFPCI : http:// www.cfpci.fr/les-cahiers-du-cfpci [consulté le 24/10/2017].

26. - La collection, qui compte sept titres, est disponible uniquement en ligne: http:// www.berose.fr/?-Les-Carnets-de-Berose-240- [consulté le 24/10/2017].

27. - La collection, qui compte onze titres, est disponible uniquement en ligne: http:// www.iiac.cnrs.fr/rubrique110.html [consulté le 24/10/2017]. 
28. - Voir : BARBE, Noël et TORNATORE, Jean-Louis (dir.). Les Formats d'une cause patrimoniale. Agir pour le château de Lunéville. Paris : CNRS-LAHIC/DPRPS-direction générale des Patrimoines, coll. «Les carnets du LAHIC », 2011. Disponible en ligne: http://www.iiac.cnrs.fr/article936.html [consulté le 24/10/2017]. CLAVAIROLLE, Françoise. La Borie sauvée des eaux. Ethnologie d'une émotion patrimoniale. Paris : CNRS-LAHIC/DPRPS-direction générale des Patrimoines, coll. «Les carnets du LAHIC », 2011. Disponible en ligne : http://www.iiac.cnrs.fr/article972.html [consulté le 24/10/2017]. DASSIÉ, Véronique. Des arbres au cœur d'une émotion. La fabrique du consensus patrimonial : le parc du château de Versailles après la tempête. Paris : CNRS-LAHIC/DPRPS-direction générale des Patrimoines, coll. «Les carnets du LAHIC», 2014. Disponible en ligne: http:// www.iiac.cnrs.fr/article1076.html [consulté le 24/10/2017].

29. - HEINICH, Nathalie. La Fabrique du patrimoine. Paris : Éd. de la MSH, coll. «Ethnologie de la France », 2009. Disponible en ligne : http://books.openedition.org/editionsmsh/2642 [consulté le 24/10/2017].

30. - Ead. Le Travail de l'Inventaire. Sept études sur l'administration patrimoniale. Paris : CNRS-LAHIC/ DPRPS-direction générale des Patrimoines, coll. «Les carnets du LAHIC », 2013. Disponible en ligne : http://www.iiac.cnrs.fr/article1050.html [consulté le 24/10/2017].

31. - http://terrain.revues.org/15915 [consulté le 24/10/2017].

32. - Au cours de ces dix dernières années, plusieurs numéros ont été entièrement ou partiellement consacrés à l'ethnologie : Le Patrimoine culturel immatériel ( $\mathrm{n}^{\circ} 116-117$, en 2008), Cinquante ans de recherche au ministère de la Culture et de la Communication ( ${ }^{\circ} 122-123$, en 2010), Patrimoines de l'Outre-mer ( $\mathrm{n}^{\circ} 126$, en 2012), Les nouveaux terrains de l'ethnologie ( $\mathrm{n}^{\circ} 127$, en 2012), Patrimoines, enjeux contemporains de la recherche $\left(\mathrm{n}^{\circ} 133\right.$, en 2016). Voir sur le site du ministère de la Culture et de la Communication: http://www.culturecommunication.gouv.fr/Politiquesministerielles/Recherche-Enseignement-superieur-Technologie/La-revue-Culture-et-Recherche. 33. - Il est important de noter, à ce propos, que l'ouvrage dirigé par Chiara Bortolotto, qui promeut une lecture critique de la notion, a été publié dans la collection "officielle» de la Mission, les «Cahiers d'ethnologie de la France », tandis que l'ouvrage collectif Le Patrimoine culturel immatériel. Premières expériences en France, le fut chez un éditeur privé (Actes Sud) et dans une collection, «L'Internationale de l'imaginaire », liée à une association (La Maison des cultures du monde). Voir : Le Patrimoine culturel immatériel. Premières expériences en France. Arles/Paris : Actes Sud/Maison des cultures du monde, coll. « Internationale de l'imaginaire », 2011).

34. - Cette partie a été développée dans un autre article, complémentaire de celui-ci : «L'article 55. Dix ans de mise en œuvre de la convention pour la Sauvegarde du patrimoine culturel immatériel ». Patrimoines. Revue de l'Institut national du patrimoine, $\mathrm{n}^{\circ} 12,2016$, p. 20-26.

35. - BENZAÏD, Redjem (rapport présenté par). L'Ethnologie de la France, besoins et projets. Paris : La Documentation française, 1980.

36. - BROMBERGER, Christian. " "Le patrimoine immatériel" entre ambiguïtés et overdose ». L'Homme, 1/2014, $\mathrm{n}^{\circ}$ 209, p. 143-151. Disponible en ligne: http://www.cairn.info/revue-lhomme-2014-1-page-143.htm [consulté le 24/10/2017].

37. - CIARCIA, Gaetano. Inventaire du patrimoine immatériel. Du recensement à la critique. Paris : CNRS-LAHIC/DPRPS-direction générale des Patrimoines, coll «Les carnets du LAHIC », 2007. Disponible en ligne : http://www.iiac.cnrs.fr/article800.html [consulté le 24/10/2017].

38. - BORTOLOTTO, Chiara (dir.). Le Patrimoine culturel immatériel. Enjeux d'une nouvelle catégorie. Paris : Éd. de la MSH, coll. «Cahiers d'ethnologie de la France », 2011. Disponible en ligne : http:// books.openedition.org/editionsmsh/3545 [consulté le 24/10/2017].

39. - Voir par exemple le programme de recherche en droit comparé du PCI mis en place par l'ISP (ENS Cachan) et l'Académie de la culture de Lettonie. Disponible en ligne: https:// dpc.hypotheses.org/le-projet-osmose [consulté le 24/10/2017].

40. - COMINELLI, Francesca. Métiers d'art et savoir-faire. Paris : Economica, 2015. 
41. - L'inventaire serait « une voie à rebours que cachent mal les subterfuges rhétoriques pour temporiser - «faire l'inventaire des inventaires» - ou se parer des vertus de l'interrogation « inventer son inventaire », du titre du récent colloque parisien qui s'est tenu à l'Institut national du patrimoine». TORNATORE, Jean-Louis. "L'inventaire comme oubli de la reconnaissance. À propos de la prise française de la convention pour la Sauvegarde du patrimoine culturel immatériel », communication au séminaire PCI du LAHIC, 19 décembre 2007. Disponible en ligne : http://www.iiac.cnrs.fr/IMG/pdf/JLTornatore.pdf [consulté le 24/10/2017].

42. - Les dossiers sont intégralement consultables en ligne : http://www.unesco.org/culture/ich/ fr/listes [consulté le 24/10/2017].

43. - Une version modifiée de cette exposition en ligne a été présentée en 2016 au musée départemental Albert-Kahn, grâce au travail de sa directrice, Valérie Perlès. Disponible en ligne : http://www.fems-pci.fr/ [consulté le 24/10/2017].

44. - Présentée en 2015, elle reste accessible via sa version en ligne : http://www.patrimoineimmateriel-aquitaine.org/ [consulté le 24/10/2017].

45. - Elle a donné lieu à un livre : BARBE, Noël et SEVIN, Jean-Christophe. L'Alsace Bossue en vingt objets... et quelques autres. Besançon : Sekoya, 2016.

46. - Comme le rappelle son titre et son préambule, la convention est avant tout instituée en vue de la sauvegarde du PCI. Chercher à en faire un texte dont la participation des communautés serait le but principal - alors que cette participation, pour essentielle qu'elle soit, ne constitue qu'un moyen - est une bien dérisoire tentative de «purification » de la notion, comme le fut en son temps la réduction du patrimoine ethnologique à la recherche en ethnologie de la France.

47. - Décret $n^{\circ} 2009-1393$ du 11 novembre 2009 relatif aux missions et à l'organisation de l'administration centrale du ministère de la Culture et de la Communication.

\section{RÉSUMÉS}

Venant après d'autres textes qui ont traité, entre 2006 et 2016 de la politique de l'ethnologie au ministère de la culture, cet article se veut un bilan des actions conduites au cours de cette décennie, marquée par l'épanouissement des recherches en l'ethnologie du patrimoine et par l'essor du patrimoine culturel immatériel, mais aussi, en 2010, à la fin de la RGPP, par la suppression de la mission du patrimoine ethnologique en tant que service autonome. A rebours des visions sommaires qui posent le diagnostic d'un remplacement de l'ethnologie par le patrimoine immatériel, mais aussi en opposition avec les cassandres qui ont prophétisé, de manière anticipée et à tout le moins précipitée, l'extinction de la première, ce bilan, où ne seront pas éludés les errements et les hésitations, tente de restituer l'esprit de synthèse entre patrimoine et ethnologie qui a été au cœur de ces dix années d'action. Axe majeur de ce travail, l'appréhension de la convention de l'UNESCO n'est pour autant pas dissociable des autres pans de ce travail, qu'il s'agisse des programmes de recherche, de l'action territoriale, ou, bien trop tardivement il est vrai, de la remise à plat de la stratégie des publications.

Coming after other texts that dealt, between 2006 and 2016, the politics of ethnology at the Ministry of Culture, this article is a summary of the actions carried out during this decade, marked by the flourishing of research in the ethnology of heritage and the rise of intangible cultural heritage, but also, in 2010, at the end of the RGPP, by the abolition of the ethnological heritage department as an autonomous service. In reverse of the visions which make the 
diagnosis of a replacement of the ethnology by the intangible heritage, but also in opposition with the cassandres which prophesied, in anticipation and at least precipitated, the extinction of the first one, This assessment, where will not be evaded errors and hesitations, attempts to restore the spirit of synthesis between heritage and ethnology that has been at the heart of these ten years of action. The main focus of this work, the apprehension of the UNESCO convention is not separable from the other parts of this work, whether research programs, territorial action, or, well, too late, it is true, of the flattened strategy of publications Coming after other texts that dealt, between 2006 and 2016, the politics of ethnology at the Ministry of Culture, this article is a summary of the actions carried out during this decade, marked by the flourishing of research in the ethnology of heritage and the rise of intangible cultural heritage, but also, in 2010, at the end of the RGPP, by the abolition of the ethnological heritage mission as an autonomous service. In reverse of the summary visions which make the diagnosis of a replacement of the ethnology by the intangible heritage, but also in opposition with the cassandres which prophesied, in anticipation and at least precipitated, the extinction of the first, This assessment, where will not be evaded errors and hesitations, attempts to restore the spirit of synthesis between heritage and ethnology that has been at the heart of these ten years of action. The main focus of this work, the apprehension of the UNESCO convention is not separable from the other parts of this work, whether research programs, territorial action, or, well, too late, it is true, of the strategy of publications

\section{INDEX}

Mots-clés : patrimoine culturel immatériel, ethnologie de la France, recherche scientifique, administration, ministère de la Culture, publications scientifiques

Keywords : intangible cultural heritage, ethnology of France, scientific research, administration, ministry of culture, scientific publications

\section{AUTEUR}

\section{CHRISTIAN HOTTIN}

Conservateur en chef du patrimoine, directeur des études du département des conservateurs, institut national du Patrimoine, membre de l'IIAC (LAHIC) christian.hottin@inp.fr 